\title{
MUNIGUA, PRAENESTE E TIBUR I MODELLI LAZIALI DI UN MUNICIPIO DELLA BAETICA
}

\section{FILIPPO COARELLI}

Università di Perugia

\begin{abstract}
Il santuario a terrazze di Munigua, scavato a partire dal 1957 dall'Istituto Archeologico Germanico, costituisce un'eccezionale ripresa, in età imperiale (tardo-flavia) di modelli architettonici sviluppatisi nel Lazio in età tardo-repubblicana. I motivi di questa ripresa vanno identificati nella volontà di collegarsi idealmente al contesto laziale da parte di una città che aveva ricevuto da poco lo ius Latii. La scelta del santuario di Hercules Victor a Tibur (accanto a quello della Fortuna Primigenia a Praeneste) si spiega con la funzione che questa divinità assunse nel culto imperiale a partire da Augusto; con gli evidenti legami mitistorici con l'Ercole di Cadice; infine, con la presenza a Tivoli di un notevole gruppo di senatori spagnoli.

El santuario de terrazas de Munigua, excavado a partir de 1957 por el Instituto Arqueológico Alemán, constituye una excepcional continuación, en época imperial (tardo-flavia), de modelos arquitectónicos desarrollados en el Lazio en época tardo-republicana. Los motivos de esta continuación se identifican en la voluntad de conectarse idealmente al contexto lacial de parte de una ciudad que había recibido hacía poco el ius Latii. La elección del santuario de Hércules Víctor de Tibur (junto al de la Fortuna Primigenia de Praeneste) se explica con la función que esta divinidad asumió en el culto imperial a partir de Augusto; con los evidentes vínculos mito-históricos con el Hércules de Cádiz; en fin, con la presencia en Tívoli de un notable grupo de senadores hispanos.
\end{abstract}

Nell'ampia, ma in gran parte stanca e ripetitiva discussione sui santuari repubblicani del Lazio (COARELLI, 1987) non è stata in alcun modo recepita l'importantissima scoperta del tempio a terrazze di Munigua (fig. 1), pur avvenuta negli ormai lontani anni'50 (GRÜNHAGEN, 1957, 329-343; idem, 1959, 275-282; idem, 1978, 290-306; GRÜNHAGEN y HAUSCHILD, 1979, 301-2). Non è il caso di approfondire in questa sede le ragioni di tale lacuna, anche se esse vanno probabilmente identificate, piuttosto che nella mancanza di un'ampia e definitiva relazione di scavo, nella separatezza che caratterizza in modo ormai drammatico i vari settori delle discipline «classiche». Eppure, già al momento della scoperta, allo scavatore non erano sfuggite le strettissime connessioni che legano il santuario di Munigua agli analoghi e più antichi monumenti del Lazio: i nomi di Praeneste e di Tibur sono esplicitamente evocati, anche se poi non si spinge a fondo l'indagine in questa direzione (GRÜNHAGEN, 1957, 280; idem, 1959, 339). Ciò del resto non era possibile nell'ambito delle sommarie notizie pubblicate al momento della scoperta. E'da presumere che l'argomento verrà affrontato in pieno nel corso della pubblicazione definitiva del monumento, che speriamo prossima. Anche in base ai dati sommari oggi disponibili, è però possibile proporre alcune osservazioni ed ipotesi, che non hanno altra pretesa se non quella di inserire l'importantissimo monumento nel più ampio discorso sui santuari repubblicani del Lazio (e in genere dell'Italia centrale), alla cui comprensione esso può portare a mio avviso un contributo fondamentale.

L'edificio, già in parte conosciuto dalla metà del'700, è stato integralmente scavato da una missione del DAI a partire dal 1956. Lo scavo si è poi 


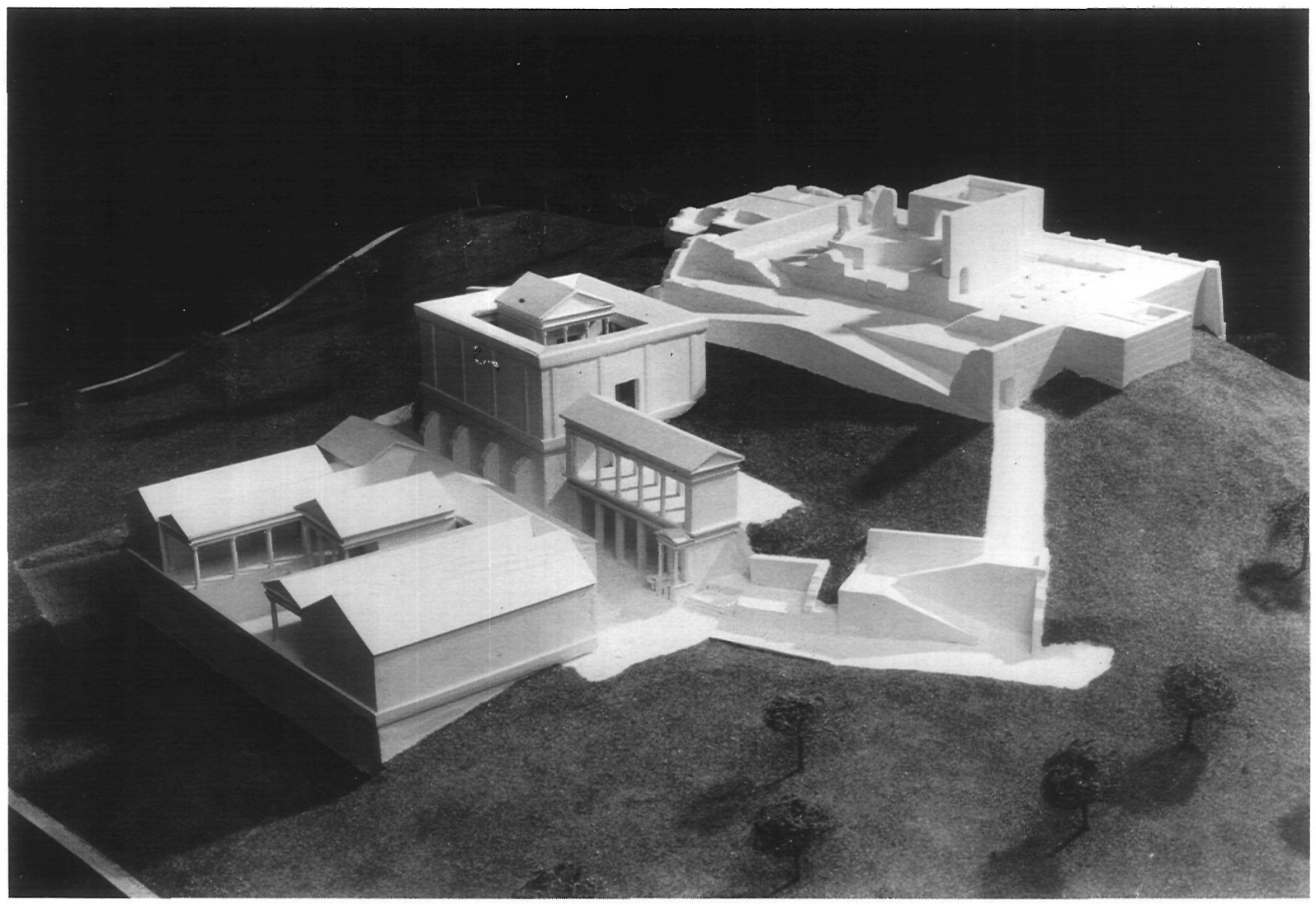

Lám. I.-Santuario di Munigua. Ricostruzione plastica. Museo Archeologico, Sevilla. Foto Instituto Arqueológico Alemán, Madrid.

esteso al resto dell'abitato e della necropoli, con risultati notevolissimi, e già in parte pubblicati (1). Proprio del primo edificio, integralmente esplorato, come si è detto, manca però ancora un'edizione definitiva: quanto si può ricavare dalle pubblicazioni finora apparse basta comunque a delineare un quadro sommario del monumento, sufficiente ai fini che qui ci si propongono.

Il santuario sorse in parte sui resti di un precedente villaggio iberico (turdetano), la cui vita sembra estendersi dal IV secolo a. C. al I d. C. (2). Si tratta di un importante terminus post quem che va ovviamente collegato con quanto sappiamo per via

(1) Sul nome e sulla scoperta, UNTERMANN, J. 1961, 10717; CARRIAZO, J. de M. 1979, 272-81. Sugli scavi, da ultimo, HAUSCHILD, TH. 1968, 358-68; idem, 1968 a, 267 ss.; idem, 1969, 185-97; idem, 1984, 159-180; VEGAS, M. 1984, 181-196; RADDATZ, K. 1973; GRÜNHAGEN, W. 1976, 226-37, Sui trovamenti epigrafici, NESSELHAUF, H., 1960, 142-54; ORS, A. D'. 1961, 203-18; FERNÁNDEZ-CHICARRO DE DIOS, C. 1972-4, 337-410. epigrafica sulla fondazione del municipio di diritto latino, che è dovuta a Vespasiano (3). La datazione sembra quindi da fissare negli anni immediatamente successivi, che vedono la nascita e lo sviluppo rapidissimo di un complesso urbano, in gran parte artificiale, che non può non collegarsi con la

(2) GRÜNHAGEN, 1957, 276-7; idem, 1959, 331. In un primo tempo si tendeva a datare il complesso all'inizio del II secolo d. C. Il villaggio iberico sembra però scomparire intorno alla metà del primo secolo d. C., e nulla giustifica uno iato cosí importante tra questo fatto e la costruzione del santuario, che sono certamente episodi connessi: la cronologia di quest'ultimo viene ora fissata dagli scavatori negli ultimi decenni del I secolo d. C.; GRÜNHAGEN y HAUSCHILD, 1979, 301; GRÜNHAGEN, 1978, 280 (datazione tardo-flavia).

(3) Sull'intervento di Vespasiano e sui municipi latini di Spagna, ELDERRY, R. K. Mc., 1918, 43-102; idem, 1919, 8694; THOUVENOT, R. 1940, 196-9; BROUGHTON, T. R. S. 1965, 126-42; SAUMAGNE, CH., 1966, 74-8; GARCÍA Y BELLIDO, A., 1967, 3-29; idem, 1972, 462-91; GALSTERER, H., 1971; SHERWIN-WHITE, A. N., 1973, 252 y 360-76; BERNARDI, A., 1973, 125-7; CASTILLO GARCÍA, C. 1975, 61417. Sul culto imperiale, ÉTIENNE, R., 1958, 447-55. 


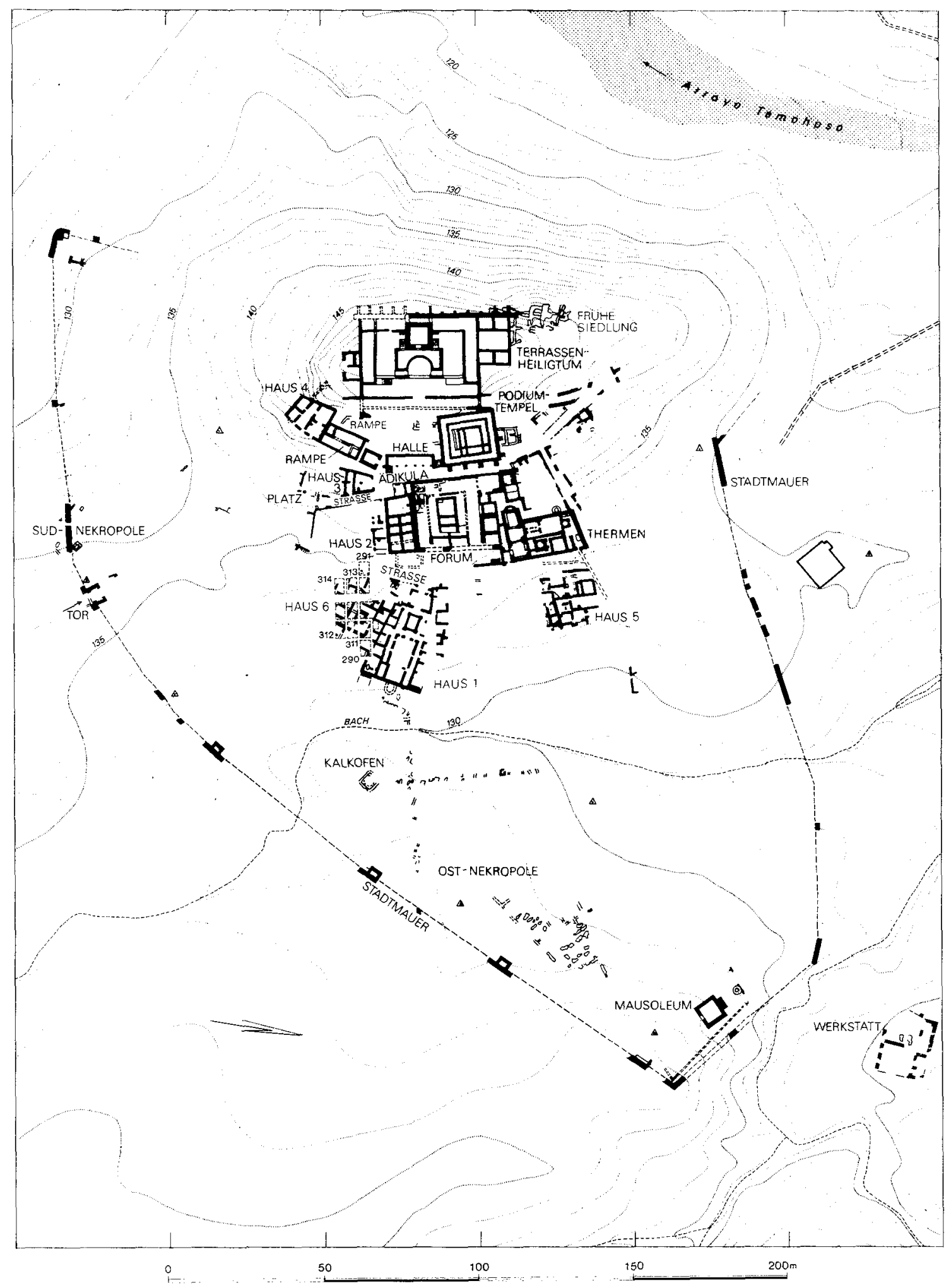

Fig. 1.-Munigua. Pianta d'insieme degli scavi (da Hauschild 1984). 


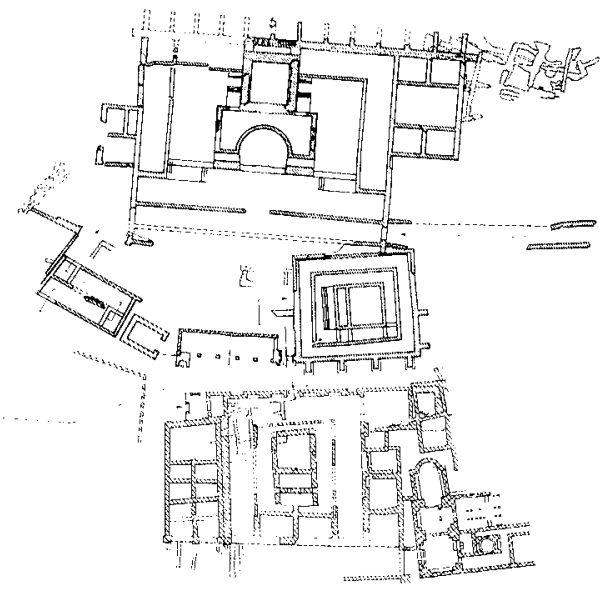

Fig. 2.-Munigua. Pianta del santuario e degli edifici adiacenti (da Raddalz, 1973).

concessione dello ius Latii. Analoghi episodi urbanistici in Spagna sono del resto largamente noti (4).

L'edificio, di dimensioni notevoli (m. $54,53 \times$ 35,20 ) (fig. $2,3,5,7$ ) occupa la sommità di un'altura che domina da ovest la città, verso la quale è orientata la sua facciata. Per ottenere un piano di imposta sufficientemente esteso fu necessario creare grandiose sostruzioni: particolarmente imponente è quella occidentale, sostenuta da poderosi contrafforti. Queste sostruzioni (a differenza dell'edificio sovrastante, completamente in laterizio) sono realizzate in opera mista, con blocchetti di pietra e duplici fasce di mattoni, distanti tra loro $60 \mathrm{~cm}$.

L'accesso al santuario consiste in una doppia rampa inclinata (larga $3 \mathrm{~m}$.) che assume l'aspetto di un grande triangolo addossato al podio del monumento. Questa conduce a una terrazza stretta ed allungata, che occupa tutta l'ampiezza dell'edificio («terrazza orientale»), ed era chiusa agli estremi nord e sud da alti muri, ma aperta al centro sul panorama sottostante.

Da qui, tramite due scalinate laterali, si saliva alla terrazza superiore, la più importante, più alta di 2,10 m. Questa è divisa in due settori dal tempio, che ne occupa il centro, ed è chiusa sui lati nord, sud e ovest da un portico sopraelevato di $60 \mathrm{~cm}$., al quale si accedeva di nuovo tramite due scale late-

(4) Si veda, ad es., la richiesta a Vespasiano della città di Sabora per poter essere ricostruita in piano: CIL II 1423; ETIENNE, 1958, 451; SORACI, R., 1982, 437 (cfr., su Munigua, p. 435). Si veda ora la grande legge municipale epigrafica di età domizianea di Irni: CRAWFORD, in JRS, 1987.

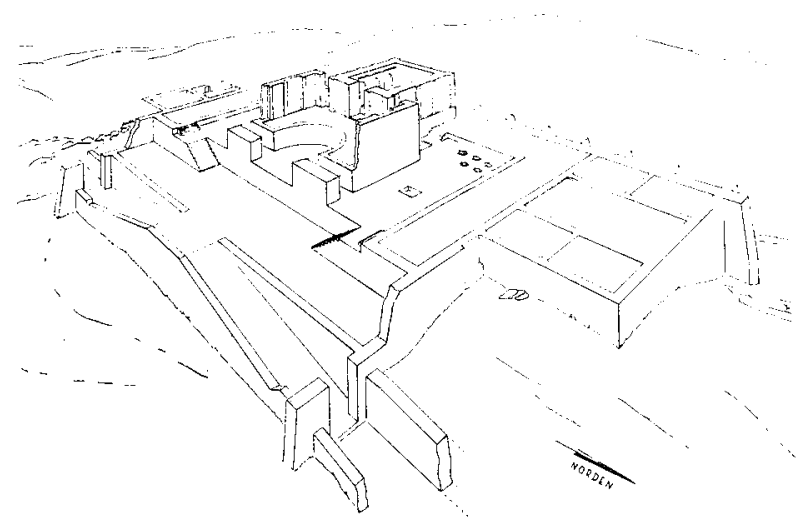

Fig. 3.-Munigua. Visione prospettica del santuario (da Grünhagen 1959).

rali, prolungamenti delle precedenti. Due analoghe scalette si trovano sul fondo, ai lati del tempio. Dei portici restano solo frammenti di archi e mattoni semicircolari e ad arco di cerchio: questa circostanza

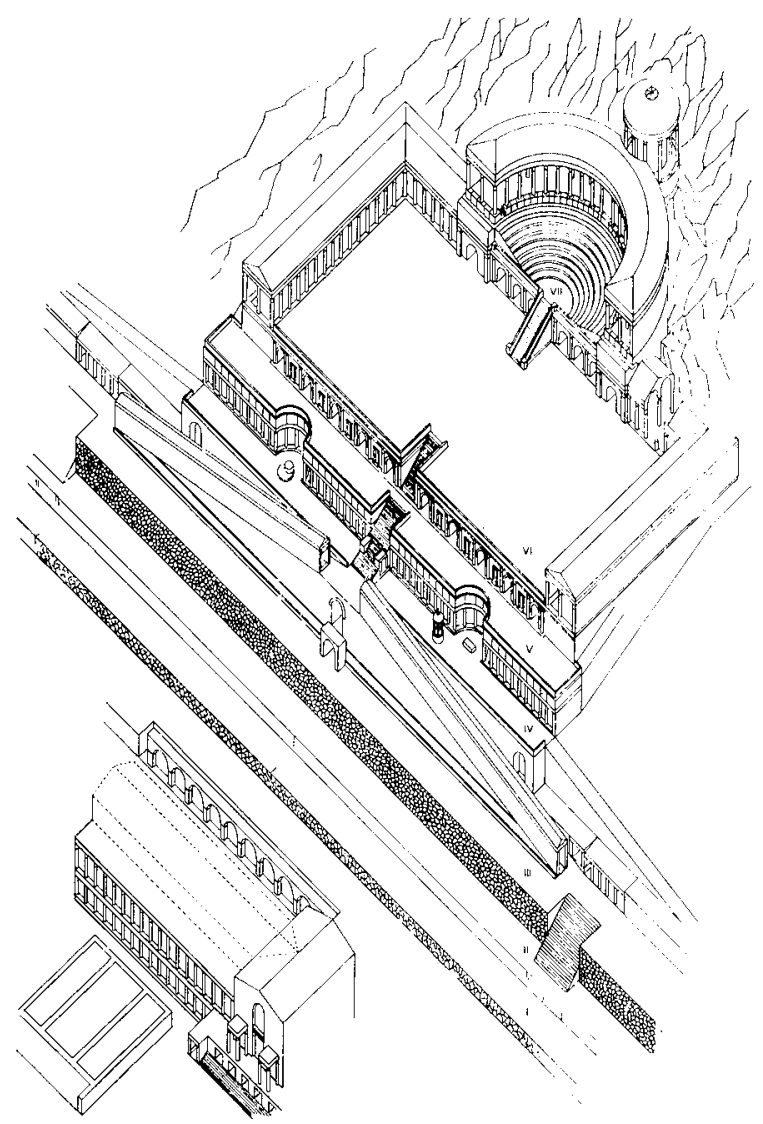

Fig. 4.-Palestrina. Il santuario della Fortuna Primigenia. Visione prospettica (da Kähler). 


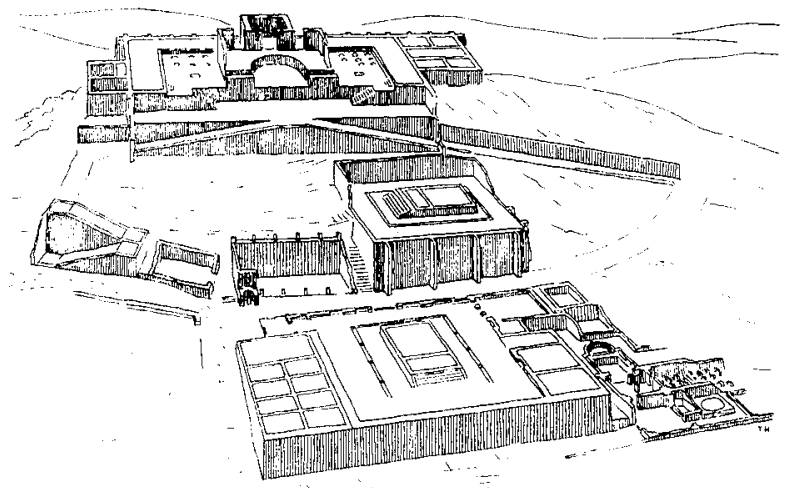

Fig. 5.-Munigua. Visione prospettica del santuario (da Hauschild 1968).

fa pensare a un portico costituito da pilastri con semicolonne addossate (piuttosto che a colonne libere).

Le acque di scolo venivano evacuate da due canalette, disposte sui lati nord e sud della terrazza. Ad un impianto idraulico appartengono anche le due vasche rettangolari che occupano il centro dei due settori della spianata. Sono di grande importanza, come vedremo, anche i grandi cercini di pietra che, in varie file parallele, occupavano il settore ovest della terrazza.

Due stretti passaggi a volta permettevano di accedere a un'esedra semicircolare aperta a est, disposta davanti al tempio, il quale sorgeva sul livello

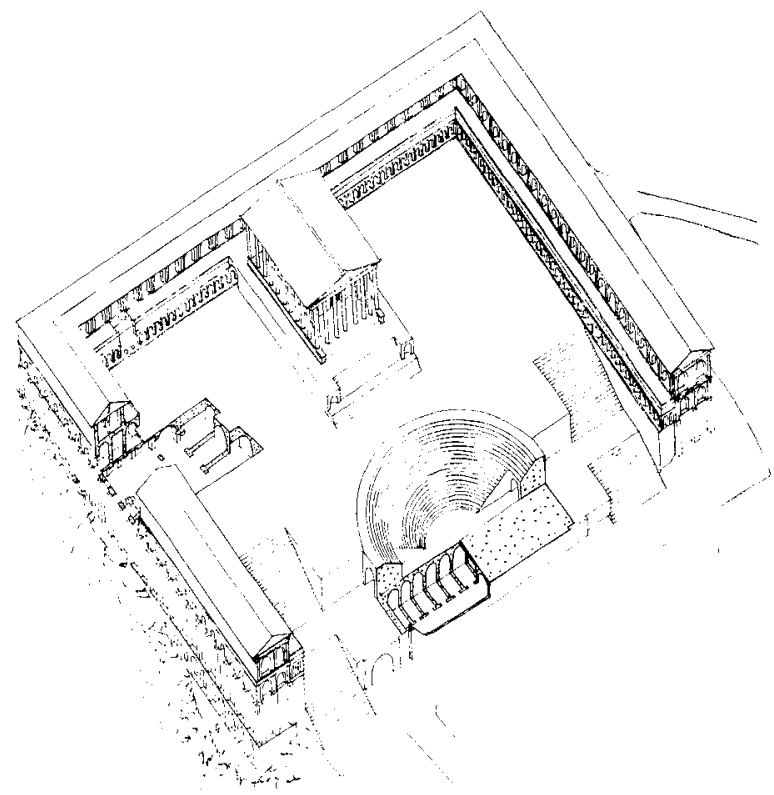

Fig. 6.-Tivoli. Santuario di Hercules Victor. Assonometria (da Giuliani 1970). soprastante, $2,10 \mathrm{~m}$. più in alto. La terrazza con fronte a semicerchio che ne risultava veniva così a costituire una sorta di pronao per il tempio stesso, ambiente chiuso sui lati da muri, in cui si aprivano due nicchie piatte, inquadrate da pilastri. L'accesso a questo, che è il settore culminante del santuario, avveniva tramite due scalinate posteriori, affiancate alla cella. Questa, in parte conservata, presentava tre nicchie poco profonde $(30 \mathrm{~cm}$.) per lato.

L'edificio era completato da due costruzioni disposte a sud e a nord: la prima era una sorta di cisterna o ninfeo in cui venivano a versarsi le acque provenienti dalla terrazza superiore. La seconda, più ampia, consisteva di due gruppi di due ambienti ciascuno, affiancati a un cortile centrale: si è pensato di identificarvi la dimora dei sacerdoti o depositi di oggetti di culto.

Da questa sommaria descrizione, e dalle piante e ricostruzioni grafiche emerge irresistibile il confronto con i santuari laziali di età repubblicana: non si tratta in alcun modo di un parallelismo generico, ma certamente di una derivazione diretta da modelli precisi, perfettamente riconoscibili — come nota l'editore del monumento- in quelli della Fortuna Primigenia a Praeneste (fig. 4) e di Hercules Victor a Tibur (GRÜNHAGEN, 1957, 280; idem, 1959, 339) (fig. 6).

Al primo rimanda senza alcun dubbio il caratteristico sistema della doppia rampa addossata, come pure l'esistenza della «terrazza orientale», che riproduce senza possibilità di dubbio (proprio per l'unicità di questa soluzione, che a Praeneste risponde a necessità funzionali del tutto peculiari) la cosiddetta «terrazza degli emicicli» (COARELLI, 1987; FASOLO y GULLINI, 1953).

Notevolmente diverso rispetto a quello di Praeneste è l'aspetto della terrazza superiore. In primo luogo, il triportico è qui sollevato rispetto all'area racchiusa, e sembra risolto con un ordine ad archi inquadrati da pilastri con semicolonne: ambedue queste caratteristiche (sopraelevazione e ordine) si ritrovano nel santuario di Hercules Victor a Tivoli, al quale rimandano anche le caratteristiche del tempio, rettangolare e inserito nel portico, e non circolare e tangente rispetto a quest'ultimo, come a Praeneste (GIULIANI, 1970, 164-201, n. 103). La presenza dell'esedra semicircolare è spiegabile come allusione in scala ridotta alle cavee teatrali dei santuari laziali (HANSON, 1952). Ma essa assume probabilmente un significato ancora più perspicuo. Come ha notato giustamente il GRÜNHAGEN (1957, 


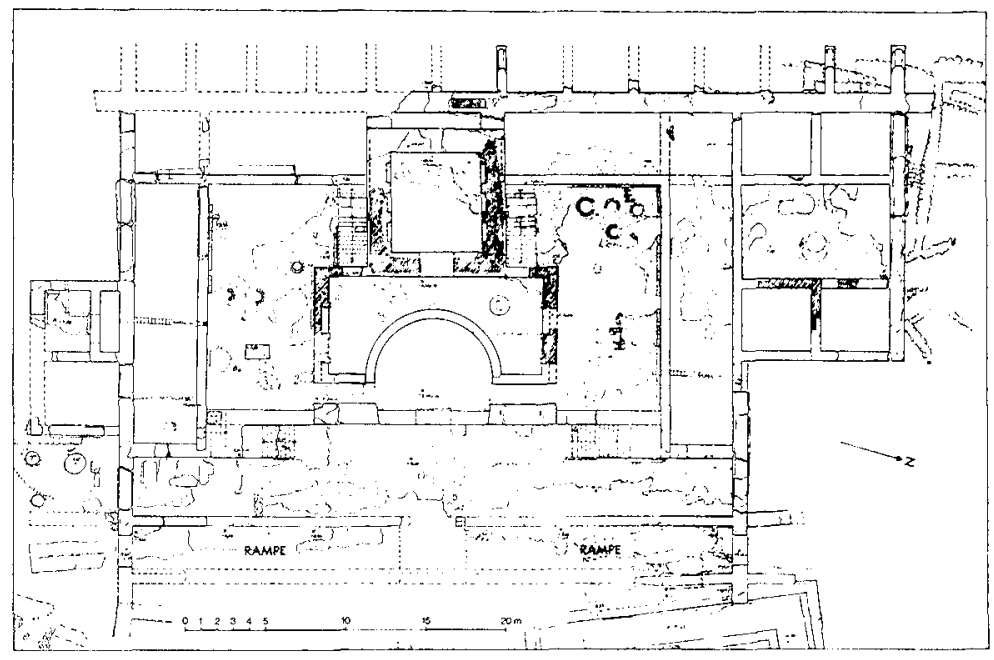

Fig. 7.-Munigua. Pianta del santuario (da Grünhagen 1978).

279), l'aspetto dell'edificio richiama il Tempio di Vespasiano a Pompei (fig. 9): ritroviamo in quest'ultimo, in particolare, la breve terrazza antistante, che costituisce una sorta di pronao, al quale danno accesso le due scalette posteriori (DE VOS y LA ROCCA, 1976, 118-121). L'identità e la contemporaneità dei due edifici difficilmente potrà ascriversi al ca-

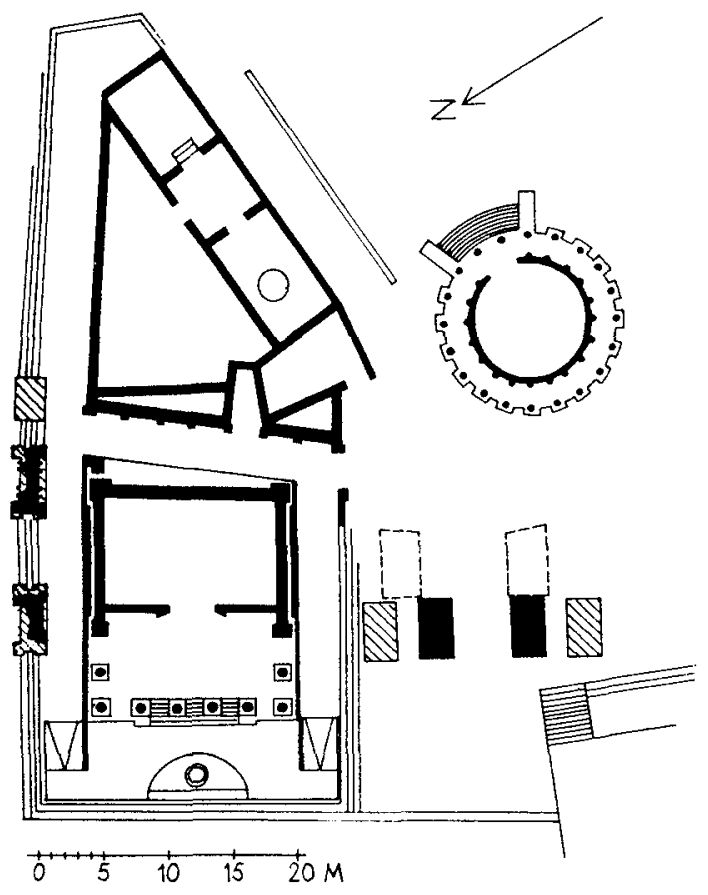

Fig. 8.-Roma. Pianta del tempio del Divo Giulio e degli edifici adiacenti (da Coarelli 1985). so. Ma è possibile anche risalire a quello che dovette costituire il modello di ambedue.

Trattandosi, nel caso del tempietto pompeiano, di un santuario del culto imperiale, è difficile sfuggire alla suggestione di un confronto con il più antico prototipo del genere: il Tempio del Divo Giulio a Roma (COARELLI, 1985, 230-232 e 307-324)

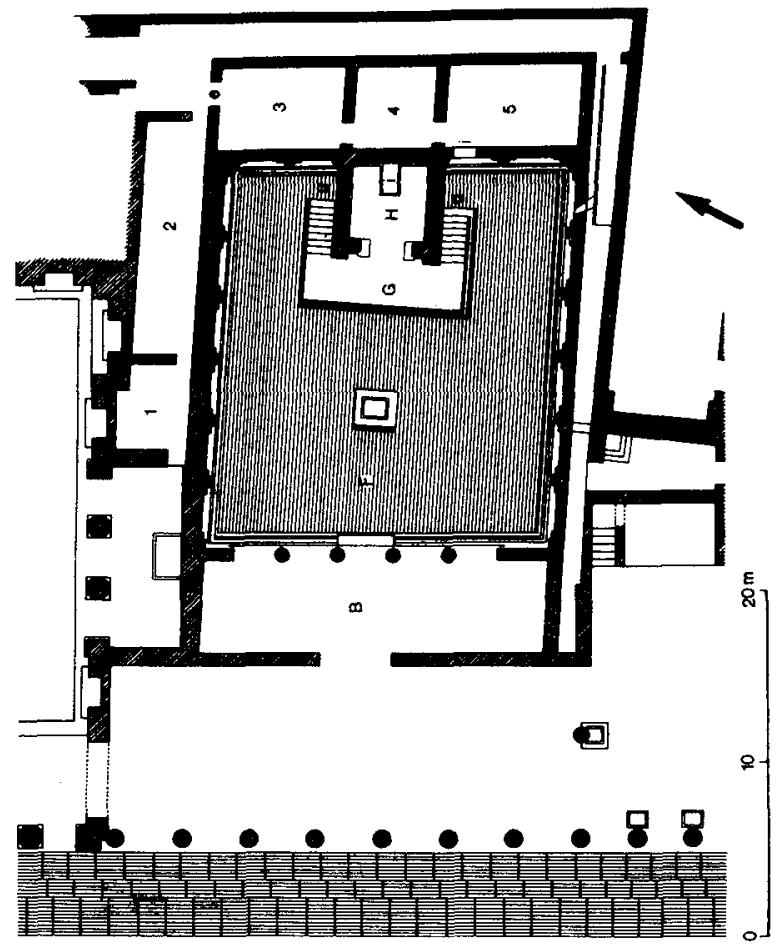

Fig. 9.-Pompei. Pianta del tempio di Vespasiano (da De VosLa Rocca 1976). 
(fig. 8). Indagini recenti hanno permesso di precisare l'aspetto dell'edificio, costituito in sintesi da un podio, aperto anteriormente in una esedra, alle spalle della quale sorgeva la vera e propria aedes. Anche in questo caso si accedeva alle strutture superiori a mezzo di due scale disposte posteriormente, sui lati del podio. L'identità della soluzione è evidente, e per quanto riguarda l'esempio di Munigua questo confronto permette anche di spiegare la presenza dell'esedra frontale, del tutto analoga al modello romano.

Se le tipologie edilizie hanno un senso, sembra inevitabile la conclusione che ci troviamo di fronte a un modello architettonico elaborato in funzione del culto imperiale.

In conclusione, il complesso di Munigua sembra costituire una sorta di incrocio, che combina in sé le principali caratteristiche dei due più importanti santuari laziali, aggiungendo a queste una connotazione particolare, legata al culto imperiale. Come è evidente, un procedimento del genere non può che corrispondere a precise funzioni ideologiche, che sarà necessario ora esplorare, uscendo dall'ambito più specificamente architettonico. Fortunatamente, $\mathbf{i}$ dati disponibili sono sufficienti per delineare una proposta di spiegazione coerente in tutte le sue parti.

In primo luogo, occorre però sottolineare un punto preliminare e determinante: se, come appare innegabile, il tempio di Munigua non è altro che una riproduzione ridotta dei due celebri santuari laziali, ogni tentativo di spiegazione dovrà confrontarsi necessariamente con il problema del grande scarto cronologico che separa questi modelli dal loro tardo epigono: in pratica, quasi due secoli. La difficoltà è aggravata dal fatto che nell'arco di questo lungo periodo mancano quasi totalmente testimonianze di tal genere. In altri termini, non è possibile ricostruire una catena continua che colleghi le realizzazioni più antiche con quest'ultima, tarda testimonianza. Ciò può significare solo che siamo in presenza di una esplicita e cosciente ricostruzione, di una vera e propria citazione «antiquaria». Corollario di questa affermazione è che il carattere «ideologico», già verificato, ne risulta notevolmente accentuato.

Le prime testimonianze che occorre mettere sul tappeto sono quelle epigrafiche. Per fortuna, gli scavi recenti hanno notevolmente arricchito lo scarso patrimonio delle iscrizioni muniguensi, e permettono ormai di ricostruire con un certo lusso di dettagli la storia amministrativa e cultuale della città.
In particolare, sufficientemente ampia è oggi la documentazione sui culti: conosciamo dediche a $\mathrm{Ce}$ res Augusta da parte di una flaminica divarum; a Fortuna Crescens Augusta, a Hercules Augustus, a Mercurius (probabilmente Augustus); a Pantheus Augustus, oltre che a Bonus Eventus e a Dispater. (FERNÁNDEZ CHICARRO, 1972-74, 337-410, fig. 1-7).

E'evidente, anche da questa semplice enumerazione, la prevalenza assoluta di divinità legate al culto imperiale. Tra queste, ci interessano naturalmente soprattutto Fortuna e Hercules: ora, va sottolineato che le iscrizioni relative provengono ambedue dal santuario a terrazze (GRÜNHAGEN, 1957, 281-2; idem, 1959, 340). La prima è stata scoperta in uno degli ambienti dell'appendice settentrionale, mentre la seconda proviene del santuario stesso, che doveva quindi comprendere tra i suoi culti quelli di Fortuna e di Ercole.

E'difficile che questa coincidenza tra le testimonianze epigrafiche e $\mathrm{i}$ dati desunti dall'analisi archeologica possa essere casuale: la scelta dei modelli architettonici sembra dunque motivata dai contenuti cultuali. Allo stesso tempo, la forma particolare del tempio, derivata da tipi edilizi utilizzati in genere per il culto imperiale, è perfettamente comprensibile nel caso di un Hercules Augustus e di una Fortuna Augusta. Occorre ora esaminare un po'più davvicino i motivi di questa scelta.

Poco si può dire di Fortuna Crescens Augusta. L'epiteto Crescens è noto solo per Iuppiter (TLL, Nomina propria II, c. 702) e si riferisce chiaramente, come si ricava dalle rappresentazioni monetali che ce ne hanno lasciato testimonianza, a Iuppiter puer. Questo, come è noto, era venerato soprattutto a Praeneste, accanto a Fortuna: si può pensare quindi che l'epiteto sia di origine prenestina. Esso del resto appare solo in monete della metà del III secolo d. C., quando il culto di Praeneste sembra conoscere una certa ripresa (5).

Assai più notevole invece la presenza di Hercules Augustus. E'evidente, in primo luogo, l'influsso dell'Hercules Gaditanus, la cui importanza per l'origine stessa del culto imperiale è stata già più vol-

(5) Monete di Gallieno: MATTINGLY y SYDENHAM, 1927, 70; di Valeriano II: ibid. pp. 35, 116-8, tav. IV 65. Nella moneta è rappresentato lo Zeus fanciullo cretese, a cavallo di una capra: è ovvia l'associazione di questo con lo luppiter Puer prenestino, e quindi con la Fortuna Primigenia. 
te sottolineata (6). In particolare, va ricordato lo stretto rapporto che con la divinità intrattennero gli imperatores romani, da Fabio Massimo Emiliano a Cesare (GAGÉ, 1940, 426). Lo stesso ritorno di Augusto dal suo grande viaggio occidentale fu assimilato al viaggio di Eracle con i buoi di Gerione dalla Spagna all'Italia (HORAT., Carm. III, 14; GAGÉ, 1940, 432). Ma questa stessa osservazione ci riporta immediatamente all'Ercole italico: l'Hercules Invictus dell'A ra Maxima (BAYET, 1926) e l'Hercules Victor italico, il cui principale centro era il celebre santuario tiburtino (HALLAM, 1931, 276-281; VERZÁR, 1985, 295-323).

L'utilizzazione di quest'ultimo come modello architettonico del santuario di Munigua ci è già parsa evidente: è in questa direzione dunque che l'indagine si presenta più promettente.

La funzione del tempio tiburtino di Hercules Victor in età imperiale ci è sufficientemente nota: a partire da un momento non precisabile, ma comunque compreso tra la fine del regno di Augusto e quello di Tiberio il collegio locale degli Herculanei si trasformò in quello degli Herculanei Augustales (SCHILLING 1942, 31-57; RE VI, A1, c. 829). L'antica divinità tiburtina venne dunque abbastanza per tempo associata al culto imperiale. Ciò si deve forse attribuire a un preciso interesse da parte di Augusto (di cui sappiamo che soleva render giustizia nei portici del santuario tiburtino) (SUET. Aug. 72; COARELLI, 1987). Particolarmente interessante è la presenza a Tivoli di numerosi personaggi che sembrano di origine spagnola (SYME, 1971, 790-2, 1035; idem, 1982-3, 241-63). E'possibile in parte collegare questo fatto con il trasferimento della corte di Adriano nella villa tiburtina, che sembra coincidere con un notevole rifiorire del culto, del resto abbastanza naturale, se si considera l'origine dell'imperatore. Tutto ciò sembra poter costituire un primo livello di spiegazione per quanto riguarda il santuario di Munigua, nella cui architettura è stato possibile riconoscere l'ispirazione tanto al modello tiburtino, quanto a edifici del culto imperiale. La presenza di una dedica ad Hercules Augustus è perfettamente coerente con tali presupposti. Il culto di Ercole come culto imperiale sembra inoltre diffondersi particolarmente tra Galba e Adriano, nel pe-

(6) TAYLOR, L. R., 1931, 163-4; GAGE, J. 1940, 425-38; ETIENNE, R. 1958, 470-1; GARCIA Y BELLIDO, A., 1963, 136-46; in generale, JACZYNOWSKA, M., 1981, 631-61 (con bibl. prec.) riodo cioè in cui fu costruito il tempio di Munigua. (JACZYNOWSKA, 1981, 645) Ma tutto ciò non basta per chiarire le ragioni che spinsero ad adottare, dopo più di un secolo e mezzo di intervallo, un tipo edilizio di età repubblicana, diffuso quasi esclusivamente in Italia centrale. Se in qualche modo ci è noto un rapporto con Tivoli, resterebbe da spiegare il collegamento altrettanto evidente con Praeneste.

In ogni caso, è inevitabile riconoscere che le ragioni di questa scelta non possono esser state superficiali ed estrinseche, dal momento che si dovette far ricorso a modelli lontanissimi - nel tempo e nello spazio- dalle soluzioni architettoniche correnti. Ancora una volta, si deve ribadire che solo profonde motivazioni ideologiche possono render conto di una soluzione così singolare. La soluzione potrà dunque venire solo dall'esame delle caratteristiche d'insieme (sociali e politiche) che presiedettero alla fondazione del Municipium Flavium Muniguense.

Come è noto, il governo di Vespasiano coincise per la Spagna (e in particolare per la Baetica) con una fase di intensissima romanizzazione, che si manifestò soprattutto attraverso la concessione dello ius Latii all'intera penisola, come risulta da un noto passo di Plinio e da numerosissime testimonianze epigrafiche (PLIN., N. H. III, 30). La colonizzazione di diritto latino si tradusse naturalmente in una urbanizzazione più o meno forzata, della quale possediamo testimonianze impressionanti: uno dei casi più notevoli è proprio quello di Munigua. L'editto originario, emanato da Vespasiano, ci è pervenuto attraverso più redazioni: da quelle note da tempo di Malaca e Salpensa a quella, recentissima e di eccezionale completezza, di Irni (vid. nota 4). Lo ius Latii (nelle due forme del Latium minus e del Latium maius) costituì fin dall'età repubblicana un potente strumento per la romanizzazione dell'Impero, e in pratica una tappa intermedia per l'acquisizione della piena cittadinanza romana. Accanto all'aspetto giuridico e formale era inevitabile che emergessero anche complesse motivazioni ideologiche, a giustificazione e convalida del nuovo diritto, che assimilava in pratica i nuovi venuti ai prisci Latini (SYME, 1971, 792).

E'probabile del resto che la concessione dello ius Latii abbia provocato (come nel caso dell'ammissione al senato dei Galli) una certa opposizione a Roma, tra i senatori di origine italica. Questa tensione ci è nota - proprio in rapporto con la Spagnada altre fonti di età imperiale (GELL. XVI, 13, 4; 
SYME, 1971, 798-9). Le lontane ascendenze italiche di molti senatori spagnoli dovettero venir riaffermate con forza, ricostruendo genealogie più o meno autentiche: è il caso degli stessi Traiano e Adriano, se almeno si può dar fede ad alcuni indizi, che sembrano condurre in questa direzione (SYME, 1971, 792, 1036).

Forse è questa la chiave d'interpretazione decisiva per spiegare il caso di Munigua: la concessione dello ius Latii e la costituzione del municipio (contemporanea, dal momento che ci rimane la menzione di quest'ultimo in una dedica a Vespasiano censore e in un'altra a Tito) (CIL II 1050; 1049) sarebbero stati in qualche modo convalidati attraverso la costruzione di un tempio che ripeteva fedelmente le forme dei due più celebri santuari del Latium Vetus, collegandolo contemporaneamente al culto imperiale: l'affermazione della latinità del municipio sarebbe in tal modo passata attraverso una riesumazione di antichi modelli architettonici, a tutti noti e riconoscibili (7).

Una conferma di tale ipotesi può venire, a mio avviso, da un'osservazione del SYME (1971; 1982 3 ), già ricordata in precedenza: Ia presenza cioè di un numero notevole di Spagnoli a Tivoli a partire dal regno di Vespasiano.

I più interessanti tra questi, nel nostro caso, sono P. Manilius Vopiscus (forse figlio del M. Manilius Vopiscus, cos. suff. nel 60 d. C.) $\left(\mathrm{PIR}^{2} \mathrm{~V} 2\right.$, 158 , n. 140, 141), amico di Domiziano, la cui villa tiburtina, prossima alle cascate dell'Aniene, fu celebrata da STAZIO (Silv. I, 3). Il probabile figlio di questi, P. Manilius Vopiscus Vicinillianus, console ordinario nel $114\left(P I R^{2} \mathrm{~V} 2,292\right.$, n. 619-620), è anch'egli ricordato in un'iscrizione di Tivoli, dalla quale sappiamo che fu curator fani Herculis (CIL XIV, 4242). L'origine spagnola, resa probabile dalla tribù, la Galeria, è stata proposta con molta verosimiglianza dal SYME (1971, 602, not. 5).

Conosciamo poi un L. Minicius Natalis, di Barcino, cos. suff. nel 106, anch'egli noto a Tivoli, con suo figlio, da varie iscrizioni. Il figlio fu patronus di Tivoli e curator fani Herculis $\left(P^{2} R^{2}\right.$ V2, $\mathrm{n}$. 619-620).

Di origine spagnola è anche, con tutta probabilità, M. Accenna Saturninus, della tribù Galeria, che fu proconsole della provincia Baetica, e anch'egli

(7) E' significativa la presenza a Munigua di case ad atrio (cfr. nota 1). è ricordato da un'iscrizione tiburtina $\left(P I R^{2} \mathrm{I}, \mathrm{n}\right.$. 14; $C I L$ XIV, 3585). Il nome ha fatto pensare a una provenienza della famiglia dall'Etruria (SYME, 1971, 1035).

Il più interessante è però il caso di L. Cornelius Pusio, cos. suff. in un anno ignoto, sotto Vespasiano. L'origine spagnola di questi è dimostrata non solo dalla tribù (la solita Galeria) ma anche dalla scoperta di un'iscrizione presso Cadice (PIR ${ }^{2}$ II, 352-3, n. 1425; DESSAU, 1902, 145-7; EE 9, 214). Egli possedeva una villa presso Tivoli (alle Acque Albule), come risulta da un'altra epigrafe (NS 1914, 101; Inscript. Italiae IV, 1, 107). Di lui possediamo probabilmente anche il ritratto in bronzo, databile a un periodo anteriore al consolato, verosimilmente in età neroniana (BIENKOWSKI, 1892, 173-203; cfr. NS 1893, 194; CIL VI, 31706). Si tratta dunque di un personaggio che iniziò la sua carriera sotto Nerone e la terminò sotto Vespasiano. Probabilmente suo figlio è il cos. suff. del $90 \mathrm{~d}$. C., L. Cornelius Pusio, rivelato dai fasti Potentini (ALFIERI, 1948, 116 y 126; DEGRASSI, 1951, 27): si è pensato che la famiglia abbia qualche rapporto con i Cornelii Balbi di Gades (8). Sappiamo l'importanza che per questi ultimi rivestì il culto dell'Hercules Gaditanus, come testimoniano le monete (GARCÍA Y BELLIDO, 1963; RODRÍGUEZ NEILA, 1975) e la notizia di Macrobio, secondo il quale un Cornelius Balbus (non sappiamo se lo zio o il nipote) avrebbe scritto un'opera in almeno 8 libri, in cui trattava degli Herculis sacra, e in particolare delle norme rituali dell'Ara Maxima (MACROB., Sat. III, 6, 16). Evidentemente, in quest'opera l'Ercole di Cadice veniva collegato con l'Hercules Invictus di Roma.

Concludendo, L. Cornelius Pusio, console sotto Vespasiano, originario di Cadice e probabilmente imparentato con i Cornelii Balbi, possedeva una villa presso Tivoli. L'origo può spiegare un suo eventuale interesse per il culto dell'Ercole gaditano; la villa è un indizio in favore di un possibile collegamento con l'Ercole Tiburtino. Non si può escludere che lo stesso L. Cornelius Pusio possa aver rivestito la cura fani Herculis, come altri senatori di origine spagnola.

Personaggi di questo genere possono aver avuto un interesse diretto all'identificazione dell'Erco-

(8) DESSAU, H., 1902, 145-7. Sui senatori della Betica, cfr CASTILLO, C., 1982, $465-519$ (Balbi: nn. 36-7, pp. 497-9: qui bibl. prec.). Sui Cornelii Balbi, cfr. RODRÍGUEZ NEILA, J. F., 1975. 
le gaditano con l'Ercole tiburtino: la costruzione del santuario di Munigua, immediatamente successiva alla concessione dello ius Latii da parte di Vespasiano, può comprendersi anche come conseguenza di un intervento diretto di senatori, quali lo stesso Cornelius Pusio, originari della provincia, e probabilmente patroni del municipio. In mancanza di testimonianze più esplicite, si tratta per ora solo di un'ipotesi, la cui coerenza e verosimiglianza ci sembrano però dimostrate dall'indagine fin qui condotta. E'auspicabile che nuove scoperte epigrafiche, di cui il suolo di Munigua non sembra avaro, portino in futuro nuovi elementi, suscettibili di chiarire un problema, la cui importanza sarebbe difficile sottovalutare.

\section{BIBLIOGRAFÍA}

ALFIERI, N. 1948: in Athenaeum, 26.

BAYET, J. 1926: Les origines de l'Hercule romain, BEFAR 132, París.

BERNARDINI, A. 1973: Nomen Latinum, Studia Ghisleriana, Pavia.

BIENKOWSKI, R. P. 1892: L. Cornelius Pusio, MDAI (R), 7.

BROUGHTON, T. R. S. 1965: Municipal Institutions in Roman Spain, Cahiers d'histoire mondiale, 9.

CARRIAZO, J. DE M. 1979: El descubrimiento de Munigua y la espiral de oro del cerro de Montoicaz, MDAI (M), 20.

CASTILLO, C. 1975: Städte und Personen der Baetica, ANRW II, 3.

- 1982: Los senadores béticos. Relaciones familiares y socirles, in Epigrafia e ordine senatorio II, Roma.

COARELLI, F. 1985: Il foro romano. Periodo repubblicano e augusteo, Roma.

- 1987: I santuari repubblicani del Lazio, Roma.

DEGRASSI, A. 1951: I fasti consolari dell'Impero, Roma.

DESSAU, H. 1902: L. Cornelius Pusio, REA 4.

DE VOS, M. y LA ROCCA, E. 1976: Guida archeologica di Pompei, Verona.

ELDERRY, R. K. 1918: Vespasian's Reconstruction of Spain, JRS, 8.

- 1919: JRS, 9.

ÉTIENNE, R. 1958: Le culte impérial dans la péninsule ibérique d'Auguste a Dioclétien, BEFAR, 191.

FASOLO, F. y GULLINI, G. 1953: Il santuario della Fortuna Primigenia a Palestrina, Roma.

FERNÁNDEZ-CHICARRO DE DIOS, C. 1972-4: Epigrafia de Munigua, AEA, 45-7.

GAGÉ, J. 1940: Hercule-Melqart, Alexandre et les Romains, REA, 42.

GALSTERER, H. 1971: Untersuchungen zum römischen Stadtwesen auf der Iberischen Halbinsel, Madrider Forschungen 8 , Berlín.
GARCIA Y BELLIDO, A. 1963: Hercules Gaditanus, AEA, 36, 1963.

- 1967: La latinización de Hispania, AEA, 40.

- 1972: Die Latinisierung Hispaniens, ANRW I, 1972.

GIULIANI, C. F., 1970: Tibur I (Forma Italiae I, 7). Roma.

GRÜNHAGEN, W. 1957: Excavaciones del Santuario de Terrazas de Munigua, V Congreso Nacional de Arqueología, Zaragoza, 1959.

- 1959: Die Ausgrabungen des Terrassenheiligtum von Munigua, Neue Deutsch Ausgrabungen in Mittelmeergebirt und im vorderen Orient.

- 1976: Eine Weihung für Dis Pater in Munigua, MDAI (M), 17.

- 1978: Farbiger Marmor aus Munigua, MDAI (M), 19.

GRÜNHAGEN, W. y HAUSCHILD, TH. 1979: Sucinto informe sobre las Excavaciones Arqueológicas en Munigua, Noticiario Arqueológico Hispánico, 6.

HALLAM, G. H., 1931: Notes on the cult of Hercules Victor in Tibur and Neighbourhood, JRS, 21.

HAUSCHILD, TH., 1968: Ausgrabungen in Munigua, Neue Kampagnen bis 1967, AA.

- 1968a: Munigua. Die doppelgeschlossige Halle und die Ädikula in Forum gebiet, MDAI (M), 9.

- 1969: Munigua. Untersuchungen im Stadtgebiet östlich vom Forum, MDAI (M), 10.

- 1984: Munigua. Vorbericht über die Grabungen in Haus I und Haus 6. Kampagne 1982, MDAI (M), 25.

HANSON, J. A. H. 1952: Roman Theater-Temples, Princeton.

JACZYNOWSKA, M. 1981: Le culte de l'Hercule romain au temps du Haut-Empire, ANRW, XVII, 2.

MATTINGLY, H. y SYDENHAM, C. 1927: The Roman Imperial Coinage, London.

MONTENEGRO DUQUE, A. 1975: Problemas y nuevas perspectivas en el estudio de la Hispania de Vespasiano, Hispania Antiqua, V.

NESSELHAUF, H. 1960: Zwei Bronzernkunde aus Munigua, MDAI (M), 1.

ORS, A. D': 1961: Miscelánea Epigráfica, Emerita, 29.

RADDATZ, K. 1973: Mulva I. Die Grabungen in der Nekropole in den Jahren 1957 und 1958, Madrider Beiträge, 2.

RODRÍGUEZ NEILA, J. F., 1975: Los Balbos de Cádiz, Sevilla.

SAUMAGNE, CH., 1966: Le droit latin et les cités romaines sous l'Empire, Publ. Inst. Droit. Univ. París 22, París.

SCHILLING, R. 1942: L'Hercule romain en face de la réforme religieuse d'Auguste, Rev. de Phil, 3. er sér. 16.

SHERWIN-WHITE, A. N. 1973: The Roman Citizenship, Oxford.

SORACI, R. 1982: Note sull'opera legislativa ed amministrativa dell'imperatore Tito, Quaderni Catanesi, 4.

SYME, R. 1971: Tacitus (trad. it. Tacito), Brescia.

- 1982-3: Spaniards at Tivoli, Anc. Soc. 13-4.

TAYLOR, L. R. 1931: The Divinity of the Roman Emperor, Middleton.

THOUVENOT, R. 1940: Essai sur la province romaine de Bétique, BEFAR 149, París (2. a ed. 1973).

UNTERMANN, J. 1961: Zum Namen von Munigua, MDAI (M), 2.

VEGAS, M. 1984: Munigua, Haus 6. Datierende Funde aus den Räumen und aus dem Brunnen, MDAI (M), 25.

VERZÁR BASS, M. 1985: L'ara di Lucius Munius a Rieti, MEFRA 97. 